\title{
Gout tophus in transferred tendons after 33 years: A case report
}

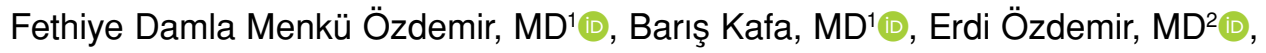 \\ Nazlı Eylem Alkanat, MDD, Ali Emre Aksu, MD'1D \\ 1Department of Plastic, Aesthetic and Reconstructive Surgery, Hacettepe University Faculty of Medicine, Ankara, Turkey \\ ${ }^{2}$ Department of Orthopedics and Traumatology, Hacettepe University Faculty of Medicine, Ankara, Turkey \\ ${ }^{3}$ Department of Pathology, Hacettepe University Faculty of Medicine, Ankara, Turkey
}

Gout is a metabolic disease due to the deposition of monosodium urate crystals in tissues, affecting $4 \%$ of the adults in the United States. ${ }^{[1]}$ Surgical intervention is one of the reasons for acute gout flares. ${ }^{[2]}$ However, there are few reports in the existing literature demonstrating surgical site gout in the long-term. ${ }^{[3-6]}$ Presence of surgical site gout in a patient without a history of gout is extremely rare and there is only a single report of surgical site gout in a case with no previous history of gout in the literature. ${ }^{[6]}$

Herein, we report an extremely rare case of surgical site gout located at the junction of the transferred tendons, flexor carpi ulnaris (FCU) tendon to extensor digitorum communis (EDC) tendon, after 33 years of the initial surgery.

Received: February 12, 2021

Accepted: March 20, 2021

Published online: November 19, 2021

Correspondence: Fethiye Damla Menkü Özdemir, MD.

Hacettepe Üniversitesi Tıp Fakültesi, Plastik, Estetik ve

Rekonstrüktif Cerrahi Anabilim Dalı, 06230 Altındağ, Ankara,

Türkiye.

E-mail: damlamenku@gmail.com

Doi: 10.52312/jdrs.2021.30

Citation: Menkü Özdemir FD, Kafa B, Özdemir E, Eylem Alkanat N. Aksu AE. Gout tophus in transferred tendons after 33 years: A case report. Jt Dis Relat Surg 2021:32(3):771-774.

(2021 All right reserved by the Turkish Joint Diseases Foundation

This is an open access article under the terms of the Creative Commons Attribution-NonCommercial License, which permits use, distribution and reproduction in any medium, provided the original work is properly cited and is not used for commercial purposes (http://creativecommons.org/licenses/by-nc/4.0/).

\section{ABSTRACT}

Surgical site gout is an extremely rare complication that is difficult to diagnose, particularly in patients without a history of gout. A 57-year-old male patient was admitted with no previous history of gout, complaining of surgical site gout located at the junction where flexor carpi ulnaris tendon was transferred to extensor digitorum communis tendon after 33 years of the initial surgery. The patient was presented with a progressive swelling over the last three months which was located on the dorsoulnar side of the right wrist joint. Magnetic resonance imaging revealed an iso/hypointense mass. During the excisional biopsy, retained non-absorbable suture materials were observed within the mass. Histopathological examination result was reported as a typical gout tophus. No recurrence was observed after 18 months of follow-up. In conclusion, surgical site gout may be observed at transferred tendons years after the initial surgery.

Keywords: Extensor digitorum communis, flexor carpi ulnaris, postsurgical, surgical site, tendon transfer, wrist.

\section{CASE REPORT}

A 57-year-old male patient was admitted to our hand surgery department complaining of a progressive swelling located on the dorsoulnar side of the right wrist joint. The patient reported that the mass was started to grow for the past three months. On physical examination, there was no tenderness or erythema around the mass. The range of motion of the wrist joint was within the normal range. Neurovascular examination findings were normal. The patient was not diagnosed with any chronic disease previously, including gout or not under any medication. He had a history of an occupational injury 33 years ago in which he lost extensor muscles and tendons on the right forearm. Thus, the patient underwent a tendon transfer surgery in which the FCU tendon was transferred to EDC tendon (Figure 1). The patient's family history was unremarkable for gout. 
Magnetic resonance imaging (MRI) revealed an iso/hypointense mass at proton density-weighted and fat-suppressed T1 images (Figure 2). We decided to perform excisional biopsy. A written informed consent was obtained from the patient. During surgery, it was confirmed that the FCU tendon was transferred to EDC tendon. The tumor was located within the junction where the FCU tendon was transferred to EDC tendon. Non-absorbable suture materials were observed within the tendon transferred region (Figure 3). A caseous material was reached following the opening of the paratenon suggestive of tendon necrosis, tuberculosis, or a foreign body reaction. An excisional biopsy was performed, and the specimens were sent for histopathological and microbiological evaluation. The weakened tendon was repaired with eight-strand and epitendinous sutures.

No organism was seen in the initial Gram staining, and there was no bacterial growth in the microbiology cultures. His preoperative blood laboratory test results were within normal ranges for complete blood count, complete metabolic panel, and uric acid levels. Microscopic examination revealed typical morphology of a gouty tophus. A granulomatous inflammation consisted of a fluffy cotton candy-like material and surrounding foreign body giant cells. At the periphery, fibrotic synovium was visible (Figure 4). Since urate crystals are dissolved in formalin fixation, microscopic examination under polarized microscope failed to demonstrate urate crystals. The patient was consulted with rheumatology department and antigout therapy was initiated. The patient's wound
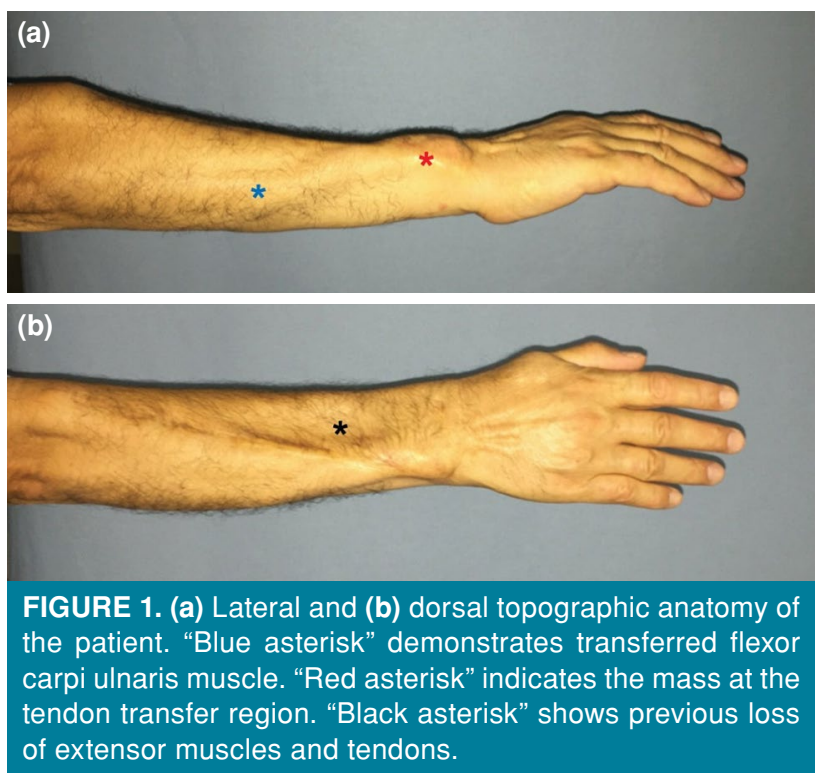

was healed without any complication. There was no recurrence at 18 months of follow-up.

\section{DISCUSSION}

Gout is a metabolic disorder caused by the chronic deposition of monosodium urate crystals in tissues. ${ }^{[7]}$ Gout tophus formation is mostly attributed to poorly controlled hyperuricemia, and mainly occurs around joints and tendons..$^{[8]}$ Gout tophus involving hand and wrist are relatively uncommon, and the vast majority of these cases present with compression of adjacent structures. ${ }^{[9,10]}$

Gout flaring is a known entity following surgery due to the catabolic events. Jeong and Jeon $^{[2]}$ reported that $44.3 \%$ of the patients developed acute gout flare during the post-surgical period in a mean 3.7-day-interval after surgery. Friedman et al. ${ }^{[11]}$ found that $33.3 \%$ of patients with a previous gout diagnosis had acute gout flare following gastric surgery. In our case, this was developed 33 years after the initial surgery, and current surgery was uneventful for acute gout flare. On
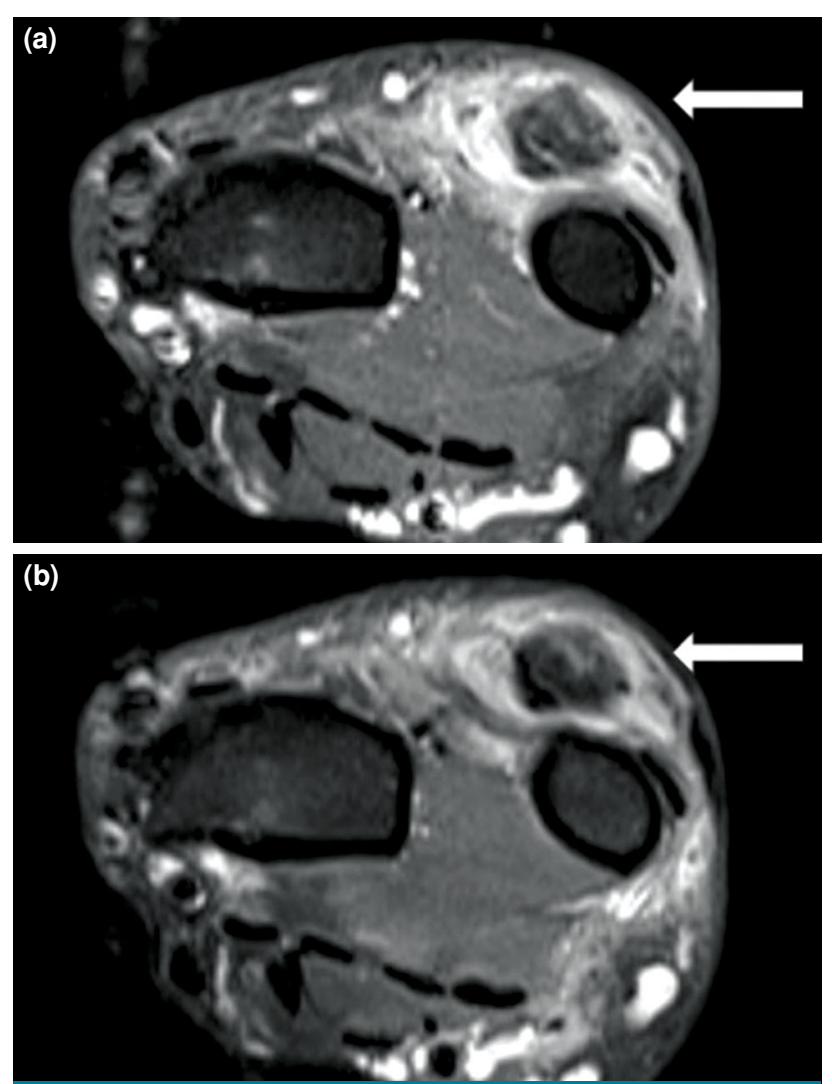

FIGURE 2. A magnetic resonance imaging scan of wrist mass. (a) Proton density-weighted and (b) fat-suppressed T1 images showing iso/hypointense mass located on the dorsal side of ulna. 

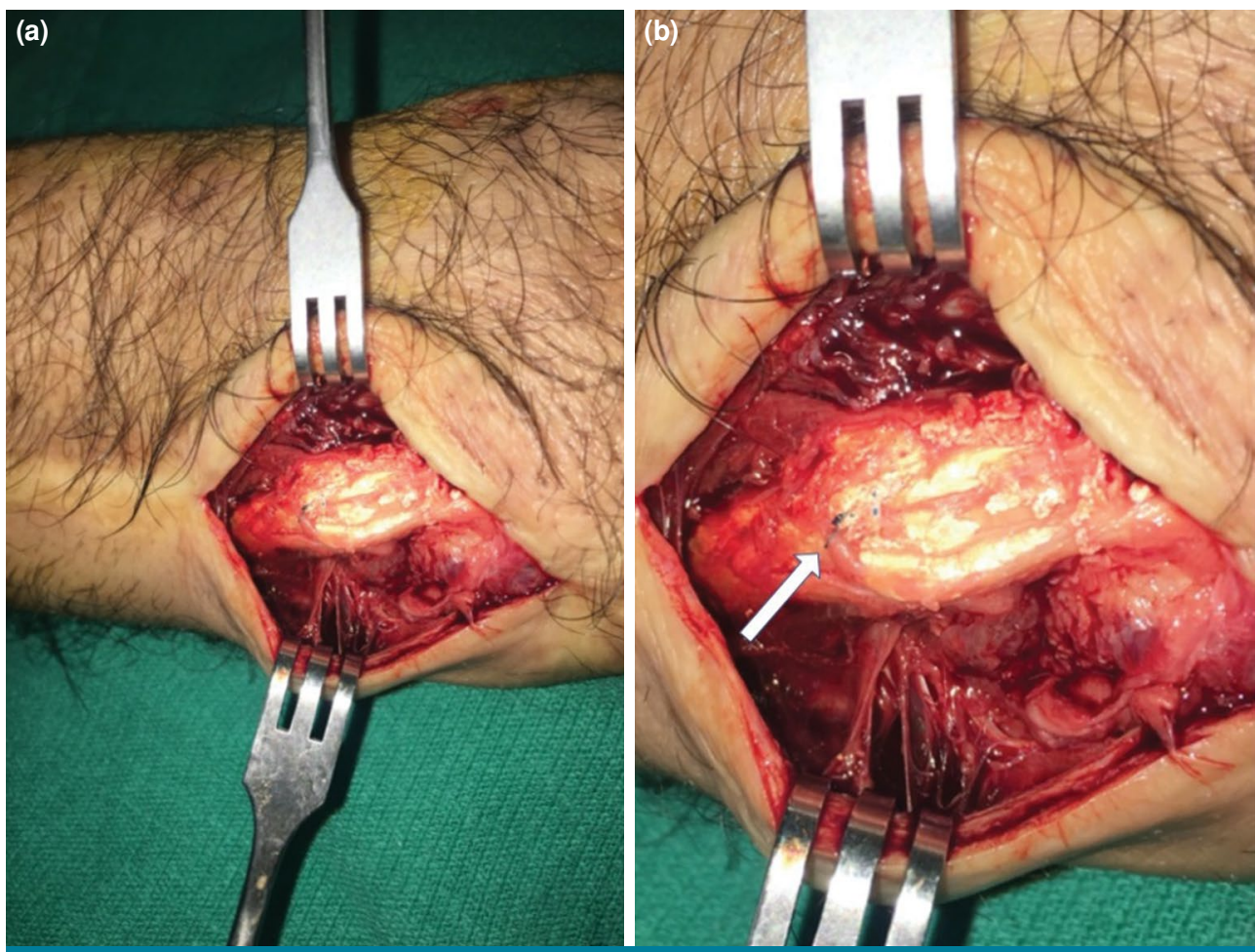

FIGURE 3. (a) An intraoperative image of gout tophus. (b) "White arrow" indicates the retained non-absorbable suture material within the mass.

the other hand, postoperative reports of gout in the long-term at the surgical site are extremely rare. Few reports have been published in the literature demonstrating surgical site gout, ${ }^{[3-6]}$ and only one of the cases was located at the

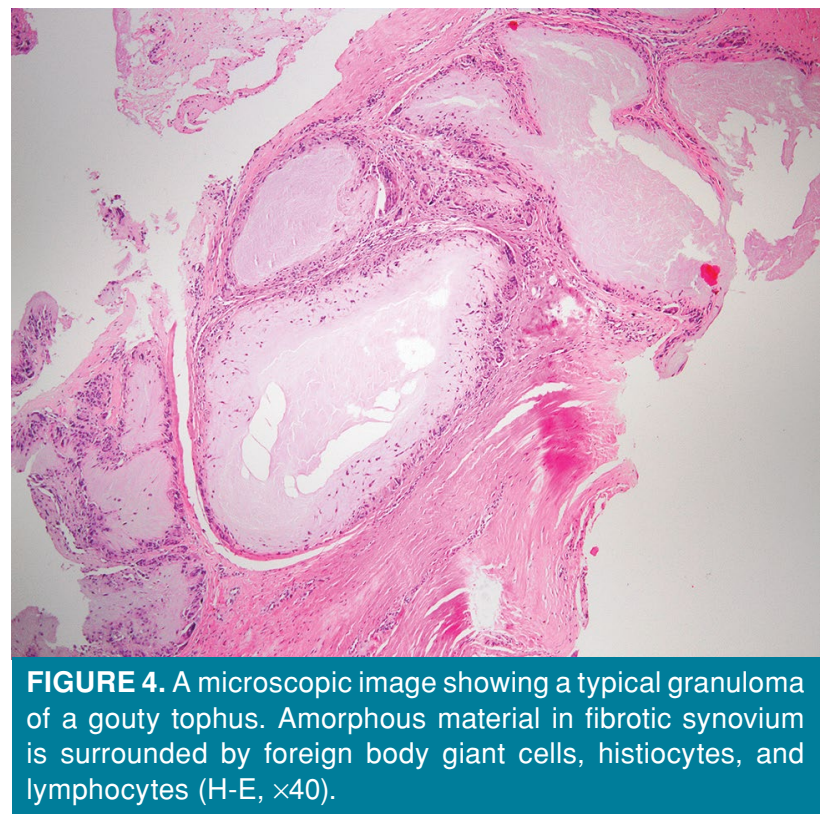

upper extremity. ${ }^{[5]}$ Ortman and Pack $^{[3]}$ reported a case with a long history of hyperuricemia who developed loosening of hip arthroplasty secondary to a gout tophus. Chen et al. ${ }^{[4]}$ reported two cases with a history of gout who had postoperative surgical site gout in 10 and 12 years after total knee arthroplasty, respectively. Giani and Gutteling ${ }^{[6]}$ reported a patient who developed surgical site gout at 12 years following an Achilles tendon repair. The unique feature of the patient was the absence of a history of gout. Sainsburry et al ${ }^{[5]}$ also reported a surgical site gout in a repaired flexor profundus tendon after seven years of surgery.

Although Wernick et al. ${ }^{[12]}$ reported that gout tophus might be the initial presentation of gout, the only case with surgical site gout was reported by Giani and Gutteling ${ }^{[6]}$ in a patient without a gout history. Our patient was not diagnosed with gout, until he developed a gout tophus at the previous tendon repair site. Uric acid level was within normal limits during the preoperative evaluation. Although hyperuricemia is a typical feature of gout, its absence does not always rule out the diagnosis. ${ }^{[7,13]}$ In our case, we used MRI to investigate differential diagnosis including malignancies. The recent literature recommends utility of both ultrasound and MRI in the diagnosis 
of gout. ${ }^{[14]}$ Although MRI finding are non-specific compared to ultrasound, it has the advantage of providing information regarding deeper structures and other potential diagnosis. ${ }^{[14]}$ The differential diagnosis of a gout tophus includes granulomatous infections originating from mycobacteria, certain types of fungi and Brucella spp., as well as sarcoidosis and foreign body reactions. ${ }^{[15]}$ Specimens obtained during excisional biopsy requires further microbiological and histological studies.

The reason for gout tophus development at the surgical site still remains unclear due to insufficient evidence in the literature. Theoretically, non-absorbable suture materials at the tendon repair site may precipitate a gout tophus, leading to mechanical friction and chronic irritation which may create an environment for the deposition of monosodium urate crystals. ${ }^{[5,6]}$ In addition, long-term retention of non-absorbable suture materials within the tissue has been reported in the literature. ${ }^{[16]}$ The gout tophus in our patient was within the junction of transferred tendons and there were non-absorbable suture materials within the mass. These findings are consistent with the mechanical friction and chronic irritation theory of Giani and Gutteling. ${ }^{[6]}$ We believe that non-absorbable suture materials and altered mechanics of tendons due to transfer surgery may precipitate gout tophi at the surgical site.

In conclusion, surgical site gout can be observed at the site of transferred tendons years after the initial surgery, despite normal uric acid levels and, therefore, it should be considered in the differential diagnosis. Non-absorbable suture materials and altered biomechanics of the tendons could be one of the reasons for surgical site gout tophus.

\section{Declaration of conflicting interests}

The authors declared no conflicts of interest with respect to the authorship and/or publication of this article.

\section{Funding}

The authors received no financial support for the research and/or authorship of this article.

\section{REFERENCES}

1. Zhu Y, Pandya BJ, Choi HK. Prevalence of gout and hyperuricemia in the US general population: The national health and nutrition examination survey 2007-2008. Arthritis Rheum 2011;63:3136-41.

2. Jeong $\mathrm{H}$, Jeon $\mathrm{CH}$. Clinical characteristics and risk factors for gout flare during the postsurgical period. Adv Rheumatol 2019;59:31.

3. Ortman BL, Pack LL. Aseptic loosening of a total hip prosthesis secondary to tophaceous gout. A case report. J Bone Joint Surg [Am] 1987;69:1096-9.

4. Chen F, Glezos C, Blum Y, Hossack M, Schwechter EM. Nonsurgical treatment of aseptic periprosthetic gout flare of the knee: A report of 2 cases. JBJS Case Connect 2016;6:e93.

5. Sainsbury DC, Hidvegi N, Blair JW. Intra-tendinous gout in a repaired flexor digitorum profundus. J Hand Surg Eur Vol 2008;33:528-9.

6. Giani VC, Gutteling E. Surgical site gout 12 years after an achilles tendon repair: A case report. JBJS Case Connect 2020;10:e1900283.

7. Ragab G, Elshahaly M, Bardin T. Gout: An old disease in new perspective - A review. J Adv Res 2017;8:495-511.

8. Sakti M, Usman MA, Lee J, Benjamin M, Maulidiah Q. Atypical musculoskeletal manifestations of gout in hyperuricemia patients. Open Access Rheumatol 2019;11:47-52.

9. Luo PB, Zhang CQ. Chronic carpal tunnel syndrome caused by covert tophaceous gout: A case report. World J Clin Cases 2018;6:279-83.

10. Doucet V, McLeod GJ, Petropolis CJ. Gouty stenosing tenosynovitis: Trigger finger as a first presentation of tophaceous gout. Plast Reconstr Surg Glob Open 2020;8:e3055.

11. Friedman JE, Dallal RM, Lord JL. Gouty attacks occur frequently in postoperative gastric bypass patients. Surg Obes Relat Dis 2008;4:11-3.

12. Wernick R, Winkler C, Campbell S. Tophi as the initial manifestation of gout. Report of six cases and review of the literature. Arch Intern Med 1992;152:873-6.

13. Atik OŞ, Ergişi Y, Ayanoğlu T, Tokgöz MA, Sezgin EA, Göçün PU. Is it easy to clinically distinguish inflammatory arthritis of bacterial origin from monoarthritis attacks of gout disease? Eklem Hastalik Cerrahisi 2016;27:167-70.

14. Davies J, Riede P, van Langevelde K, Teh J. Recent developments in advanced imaging in gout. Ther Adv Musculoskelet Dis 2019;11:1759720X19844429.

15. Kostman JR, Rush P, Reginato AJ. Granulomatous tophaceous gout mimicking tuberculous tenosynovitis: Report of two cases. Clin Infect Dis 1995;21:217-9.

16. Ergin ÖN, Demirel M, Özmen E. An exceptional case of suture granuloma 30 years following an open repair of achilles tendon rupture: A case report. J Orthop Case Rep 2017;7:50-3. 